University of Nebraska - Lincoln

DigitalCommons@University of Nebraska - Lincoln

\title{
Stochastic Analysis of Energy Consumption in Wireless Sensor Networks
}

\author{
Yunbo Wang \\ University of Nebraska-Lincoln, yunbow@cse.unl.edu \\ Mehmet C. Vuran \\ University of Nebraska-Lincoln, mcvuran@cse.unl.edu \\ Steve Goddard \\ University of Nebraska-Lincoln, goddard@cse.unl.edu
}

Follow this and additional works at: https://digitalcommons.unl.edu/cseconfwork

Part of the Computer Sciences Commons

Wang, Yunbo; Vuran, Mehmet C.; and Goddard, Steve, "Stochastic Analysis of Energy Consumption in Wireless Sensor Networks" (2010). CSE Conference and Workshop Papers. 154.

https://digitalcommons.unl.edu/cseconfwork/154

This Article is brought to you for free and open access by the Computer Science and Engineering, Department of at DigitalCommons@University of Nebraska - Lincoln. It has been accepted for inclusion in CSE Conference and Workshop Papers by an authorized administrator of DigitalCommons@University of Nebraska - Lincoln. 


\title{
Stochastic Analysis of Energy Consumption in Wireless Sensor Networks
}

\author{
Yunbo Wang, Mehmet C. Vuran and Steve Goddard \\ Department of Computer Science and Engineering \\ University of Nebraska-Lincoln \\ Email: \{yunbow, mcvuran, goddard\}@ cse.unl.edu
}

\begin{abstract}
Limited energy resources in increasingly sophisticated wireless sensor networks (WSNs) call for a comprehensive crosslayer analysis of energy consumption in a multi-hop network. For reliability analysis in such networks, the statistical information about energy consumption and lifetime is required. However, traditional energy analysis approaches only focus on the average energy consumed. In this paper, instead, we provide a stochastic analysis of the energy consumption in a random network environment. Accordingly, a comprehensive cross-layer analysis framework, which employs a stochastic queueing model, is developed. Using this framework, the distribution of energy consumption for nodes in WSNs during a given time period is found. We show that when the time duration is long, the energy consumption asymptotically approaches the Normal distribution. This distribution of energy consumption is then utilized to investigate the distribution of node lifetime and network lifetime. The developed analysis framework is generic and is parameterized for many WSN protocols, including an anycast protocol as a case study. Comprehensive simulations and testbed experiments are provided to validate the developed model. The cross-layer framework is also used to identify relationships between the distribution of energy consumption and network parameters, such as network density, duty cycle, and traffic rate. To the best of our knowledge, this is the first comprehensive work to investigate probabilistic distribution of energy consumption in WSNs.
\end{abstract}

\section{INTRODUCTION}

In most Wireless Sensor Network (WSN) applications, nodes are powered by batteries, and replacing the batteries is a tedious work. When energy is depleted, nodes become inactive, losing their sensing and communication functionalities. Therefore, to improve network reliability and prolong network lifetime, utilizing and evaluating various energy-saving techniques, such as periodic sleeping, is of great importance in network designs.

Accurately characterizing increasingly sophisticated energysaving techniques $[1,4]$ is a great challenge. In MAC layer, periodic sleeping based protocols [3, 21, 29] have been developed, where nodes are forced into sleeping mode periodically, while still maintaining network connectivity. In network layer, energyaware routing protocols $[2,8,22]$ are also utilized to further reduce the energy consumption. Complicated network activities in multiple protocol layers necessitate a comprehensive and generic model to accurately evaluate the energy consumption in WSNs.

Traditionally, energy analyses are focused on the average power consumption. For example, in studies proposing the previously mentioned energy-efficient WSN protocols, the authors show the energy efficiency of their protocols with a reduced average energy consumption than other protocols. Moreover, existing generic energy analysis models $[6,20,27]$ also focus on the average energy consumption. However, due to the random nature of the wireless environment, in critical applications where

Supported, in part, by grants from the National Science Foundation (0707975) and the Air Force Office of Scientific Research (FA9550-06-1-0375). a highly reliable network is required, only knowing the average energy consumption is insufficient. For instance, to evaluate if a network has a 95\% reliability to function more than 1 year, additional statistics about the energy consumption is required.

In this paper, we develop a Markov chain-based cross-layer analytical model to investigate the statistics of energy consumption in WSNs. Instead of the average energy consumption, we obtain the probabilistic distribution of the energy consumption, i.e., the probability that the consumed energy within any given period is lower than a specific threshold. The energy consumption distribution is a natural tool to evaluate reliabilitycritical networks. As an example, our stochastic model is used to investigate the lifetime distribution of a node and the network. Existing lifetime analysis work, such as in [9], only provides the average lifetime. Although probabilistic lifetime is investigated in $[17,18]$, these studies focus on a specific network topology and simplistic MAC protocols. In contrast, our lifetime analysis is designed to be generic and comprehensive. Protocols in both MAC layer and network layer, traffic rate, wireless channels, as well as queueing behaviors are all captured in the developed framework to reveal their effects on the energy consumption.

The contributions of this paper are as follows: First, a comprehensive and accurate cross-layer analysis framework is developed to characterize the energy consumption distribution during a given period of time in WSNs. The Markov process based framework is generic and is parameterized for many WSN protocols. Second, it is shown that when the time period is large enough, the energy consumption converges to a Normal distribution. This result greatly reduces the computation cost for the analysis. Third, the node lifetime distribution and the network lifetime distribution are derived using the energy consumption distribution, and the relationships between network parameters and the lifetime distribution are investigated. With this framework, scheduling, deployment, admission control, and communication solutions can be developed to provide probabilistic lifetime guarantees in reliability-critical applications. To the best of our knowledge, this is the first comprehensive work to investigate probabilistic energy consumption in WSNs.

The remainder of this paper is organized as follows: In Sec. II, the energy consumption distribution problem and the probabilistic lifetime problems are formally defined, and an overview of our Markovian model is provided. The detailed derivation of the single-node energy consumption distribution is developed in Sec. III. The single-node lifetime distribution and network lifetime distribution are then given in Sec. IV. In Sec. $\mathrm{V}$, a case study for a specific protocol - the anycast protocol is provided. Simulations and experimental results are given in Sec. VI to validate the developed model. Finally, Sec. VII concludes the paper, and suggests future research directions. 


\section{Problem Definition And System Model}

In our analysis, a WSN with $N$ nodes is considered. The traffic at each node $A$ consists of two parts: the locally generated traffic and the relay traffic. The arriving rate for these two types of traffic are $\lambda_{A}^{l}$ and $\lambda_{A}^{f}$, respectively. Node $A$ is also characterized by its queue length, $M_{A}$, and the battery capacity, $C_{A}$. In this paper, when there is no ambiguity, subscript $A$ in any node-specific variables is omitted. Furthermore, a lognormal fading channel model [30] is considered for wireless channel effects. Accordingly, we are interested in the following problems:

1) For any node in the network, given the traffic rates $\lambda^{l}$ and $\lambda^{f}$, the queue length $M$, and the MAC protocol, what is the energy consumption distribution in a period of time $T, F_{E(T)}(e)$ ?

2) Given the energy consumption distribution for the node, and a battery capacity $C$, what is the distribution of its lifetime, $F_{L(C)}(t)$ ?

3) Given the energy consumption distribution, and a battery capacity $C_{i}$ for each node $i$, what is the distribution of the network lifetime, $F_{N L}(t)$ ?

Of course, these random variables are dependent on the network topology and protocols. In this section, an overview of our generic and parameterized solutions for the above problems is provided. The detailed description of the framework is elaborated in Sections III-IV.

\section{A. Single Node Energy Consumption Distribution}

Each node in the network is modeled by a discrete-time queueing system with time unit $T^{u}$. and is characterized by its traffic interarrival distribution and service process. More specifically, in each time unit, the traffic interarrival is modeled according to a Bernoulli process. Furthermore, a Discrete Time Markov Process (DTMP) is used to model the service behavior. Therefore, the service time is Phase-Type ( $\mathrm{PH})$ distributed [15, Ch. 2]. We also assume that the queueing policy is First-ComeFirst-Serve.

1) Arrival Pattern: The Bernoulli arrival process is motivated by the following: In a multi-hop WSN, locally generated packets consist of the local information sampled by the sensors, regardless if the node is listening, whereas relay packets are received from the neighbors of the node, only when the node is listening.

The arrival pattern of the locally generated packets depends on the application requirements. For monitoring applications, where nodes repeatedly poll their sensors, the generated data is periodic. Accordingly, the locally generated traffic can be modeled using a constant bit rate (CBR) model. For event-based applications, nodes send data only if a certain physical event of interest occurs, e.g., the temperature exceeds a certain threshold. In this case, the generated data are sporadic or bursty. In this paper, we focus on event-based applications generating sporadic traffics. Considering that such physical events do not occur very frequently, we assume that the probability with which the event occurs at any time is governed by a Poisson process. Other types of traffics can be captured by extending our queueing model to adopt a Markov Arrival Process (MAP) [16, Ch. 5]. However, due to limited space, they are out of the scope of this paper, and will be covered in our future work. In a discrete time model with a small enough time unit $T^{u}$, the probability that more than one events occur in a time unit is negligible. Thus, the Poisson process is equivalent to a Bernoulli process [14, Ch. 6] in each time unit.
The relay traffic, however, depends on the network topology and protocols. Our experiment results reveal that, for multi-hop communications, the arrival pattern for relay traffic in either monitoring applications or event-based applications converges to a Bernoulli distribution [28]. Accordingly, the local and forwarding traffic rates $\lambda^{l}$ and $\lambda^{f}$ at a node are defined to be the probability that a new locally generated packet or relay packet arrives during a time unit $T^{u}$, respectively. When the node is not listening, input traffic rate is $\lambda^{l}$, whereas when the node is listening, the traffic rate is $\lambda^{l}+\lambda^{f}$.

2) Service Time: Since each node is modeled according to a discrete-time Markov process (DTMP) with time unit, $T^{u}$, the service time of each node is Phase-Type (PH) distributed. Because a Bernoulli arriving process is assumed for packets and the DTMP is used to describe the behavior of packet transmission service, the system is essentially governed by a Quasi-Birth-Death (QBD) process [15, Ch. 3].

The recurrent discrete-time Markov chain (DTMC) for the process, denoted as $\left\{X_{n}\right\}$, has a layered structure, as shown in Fig. 1(a). Each layer $i$ contains the part of the chain where there are $i$ packets in the queue. The communication behaviors of each node are represented by transitions among states in $\left\{X_{n}\right\}$. The detailed explanation of this DTMC is provided in Sec. III. Based on this DTMC, the pdf for the single-node energy consumption, $E(T)$, is found for any given duration $T$. It is also shown that, when $T$ is large enough, $E(T)$ asymptotically approaches the Normal distribution. The mean and the variance of such distribution are given in Sec. III-C.

\section{B. Node Lifetime and Network Lifetime Distribution}

The lifetime distribution of a node depends on the energy consumption distribution during any given period $T$, and the total capacity of its battery $C$. Moreover, the network lifetime distribution depends on the lifetime distribution for each node, and how the network lifetime is defined. For different applications and network topologies, the network lifetime should be defined differently [5]. While a complete investigation of network lifetime with various definitions is out of scope in this paper, we focus on the lifetime defined as the duration before the battery depletion of the first node. In Sec. IV, the node lifetime distribution and network lifetime distribution are found based on the single-node energy consumption distribution.

\section{Single Node Energy Consumption Distribution}

In WSNs, energy is consumed by each node for communication, sensing, computing, and other activities. While other activities are dependent on applications and hardware platforms, the communication process is common among most applications and platforms. Therefore, in this paper, only energy consumed for the communication is considered. Our Markov-chain based technique can be easily extended to account for the energy consumed in processing or sensing.

The communication behaviors of each node are represented by transitions among states in Markov chain $\left\{X_{n}\right\}$. In the following, the construction of states and transitions in $\left\{X_{n}\right\}$ is discussed. Then, the single-node energy consumption distribution is found.

\section{A. Constructing Markov chain $\left\{X_{n}\right\}$}

As shown in Fig. 1, the Markov chain $\left\{X_{n}\right\}$ is divided into a Idle layer and $M$ Transmission layers. Each layer $m(1 \leq$ $m \leq M)$ represents the transmission process when there are $m$ packets in the system, and layer 0 represents the idle process 


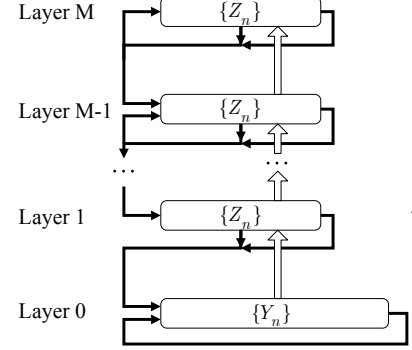

(a) $\left\{X_{n}\right\}$
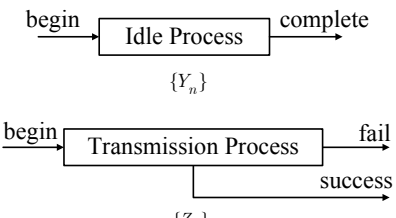

$\left\{Z_{n}\right\}$

(b) $\left\{Y_{n}\right\}$ and $\left\{Z_{n}\right\}$

Fig. 1. The layered structure of Markov chains $\left\{X_{n}\right\}$ is shown in (a). $\left\{Y_{n}\right\}$ and $\left\{Z_{n}\right\}$ are shown in (b). The structures of $\left\{Y_{n}\right\}$ and $\left\{Z_{n}\right\}$ are parameterized by the MAC protocol.

when there is no packet to be transmitted. Note that the names of transmission process and idle process do not necessarily indicate that nodes are always transmitting or idle in these processes. For example, in the idle process for a periodic sleeping protocol, nodes perform default sleeping-waking cycle, and may be in either sleeping or listening communication state.

The block structures in $\left\{X_{n}\right\}$ for the idle layer and a transmission layer are denoted as $\left\{Y_{n}\right\}$ and $\left\{Z_{n}\right\}$, respectively, as shown in Fig. 1(b). According to the MAC protocol employed, they are parameterized by the following notations.

- $\boldsymbol{P}_{Y}$ and $\boldsymbol{P}_{Z}$ : the transition probability matrices among the states in $\left\{Y_{n}\right\}$ and $\left\{Z_{n}\right\}$, respectively.

- $\boldsymbol{\alpha}_{Z}$ and $\boldsymbol{\alpha}_{Z}$ : the initial probability vector for $\left\{Y_{n}\right\}$ and $\left\{Z_{n}\right\}$, respectively. With each probability in these vectors, the idle and communication process starts in the corresponding state in $\left\{Y_{n}\right\}$ and $\left\{Z_{n}\right\}$, respectively.

- $\boldsymbol{t}_{Y}^{s}$ and $\boldsymbol{t}_{Z}^{s}$ : the probability vector from each state in $\left\{Y_{n}\right\}$ and $\left\{Z_{n}\right\}$ to complete the idle process and the transmission process successfully, respectively.

- $\boldsymbol{t}_{Z}^{f}$ : the probability vector from each state in $\left\{Z_{n}\right\}$ to complete the transmission process unsuccessfully.

- $\lambda_{Y}$ and $\lambda_{Z}$ : the packet arrival probability vector for each state in $\left\{Y_{n}\right\}$ and $\left\{Z_{n}\right\}$, respectively. They are functions of $\lambda^{l}$ and $\lambda^{f}$. For the states where the node is listening, the corresponding elements in $\lambda_{Y}$ and $\lambda_{Z}$ are $\lambda^{l}+\lambda^{f}$, otherwise the elements are $\lambda^{l}$.

Although the states and transitions within each layer, i.e., $\left\{Y_{n}\right\}$ and $\left\{Z_{n}\right\}$, is MAC protocol dependent, the interactions among layers are common for many MAC protocols, parameterized by the above transition probability matrices. Accordingly, the transition probability matrix, $Q_{X}$, of the entire Markov chain $\left\{X_{n}\right\}$ can be found using these matrices as explained next.

For layer $m, 1 \leq m \leq M-1$, the queue is not full. When a packet arrives and the current transmission is not completing, the process transits to a higher layer, since the queue length increases. The probabilities of such transitions are governed by the matrix

$$
\boldsymbol{A}_{u}=\left(\mathbf{1} \boldsymbol{\lambda}_{Z}\right)^{\mathrm{T}} \otimes \boldsymbol{P}_{Z}
$$

where $\mathbf{1}$ is a properly dimensioned matrix containing all 1's, and $\otimes$ is the entry-wise product operator. $\boldsymbol{\lambda}_{Z}$ and $\boldsymbol{P}_{Z}$ are parameterized according to the MAC protocol. Note that element $(i, j)$ in $\boldsymbol{A}_{u}$ represents the transition probability from the $i$ th state in previous layer to the $j$ th state in the upper layer, and other transition probability matrices in the following are defined the similar way. The transition probability matrix within the

same layer $m, 1 \leq m \leq M-1$, is

$$
\boldsymbol{A}_{s}=\left(\mathbf{1} \boldsymbol{\lambda}_{Z}\right)^{\mathrm{T}} \otimes\left(\boldsymbol{t}_{Z} \boldsymbol{\alpha}_{Z}\right)+\left(\mathbf{1}-\mathbf{1} \boldsymbol{\lambda}_{Z}\right)^{\mathrm{T}} \otimes \boldsymbol{P}_{Z},
$$

where $\boldsymbol{t}_{Z}=\boldsymbol{t}_{Z}^{s}+\boldsymbol{t}_{Z}^{f}$ is the probability vector from each layer to complete the current transmission process regardless of success or failure. The first term in (2) captures the case where a locally generated packet arrives at the same time unit in which a packet service is completed. The second term in (2) is for the case where neither service completion nor new packet arrival occurs during the time unit.

At layer $m=M$, the queue is full. Hence, new arriving packets are dropped. Therefore, the transition probability matrix within this layer is $\boldsymbol{A}_{u}+\boldsymbol{A}_{s}$.

If there is no packet arrival and the current packet service is completed at the current time unit, the Markov chain transits to the lower layer. The transition probability matrix from layer $m+1$ to layer $m,(1 \leq m \leq M-1)$, is

$$
\boldsymbol{A}_{d}=\left(\mathbf{1}-\mathbf{1} \boldsymbol{\lambda}_{Z}\right)^{\mathrm{T}} \otimes\left(\boldsymbol{t}_{Z} \boldsymbol{\alpha}_{Z}\right) .
$$

The transition probabilities are similar when the idle layer is involved as shown below:

$$
\begin{aligned}
& \boldsymbol{A}_{u 0}=\boldsymbol{\lambda}_{Y}^{\mathrm{T}} \boldsymbol{\alpha}_{Z}, \\
& \boldsymbol{A}_{d 0}=\left(\mathbf{1}-\mathbf{1} \boldsymbol{\lambda}_{Z}\right)^{\mathrm{T}} \otimes \boldsymbol{t}_{Z} \boldsymbol{\alpha}_{Y}, \\
& \boldsymbol{A}_{s 0}=\left(\mathbf{1}-\mathbf{1} \boldsymbol{\lambda}_{Y}\right)^{\mathrm{T}} \otimes\left(\boldsymbol{P}_{Y}+\boldsymbol{t}_{Y}^{s} \boldsymbol{\alpha}_{Y}\right) .
\end{aligned}
$$

When a new packet arrives while there is no packet in the system, the chain transits from the idle layer to layer 1 according to $\boldsymbol{A}_{u 0}$ in (4). When the service is completed for the only packet in the system and no new packet arrives, the chain transits from layer 1 to the idle layer according to $\boldsymbol{A}_{d 0}$ in (5). Finally, the transition probabilities with which the node stays in the idle layer are given in $\boldsymbol{A}_{s 0}$ in (6).

Using (1)-(6), the transition probability matrix $Q_{X}$ for the entire recurrent Markov chain $\left\{X_{n}\right\}$ is constructed as follows:

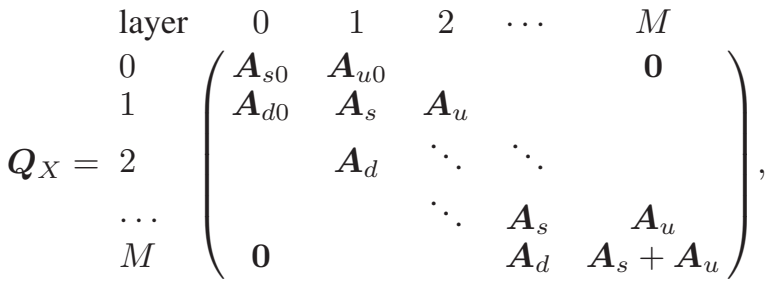

where each non-zero block corresponds to the transition probability among all layers. Then, the equilibrium state probability vector, $\pi$, for $\left\{X_{n}\right\}$ is calculated by solving $\pi Q_{X}=\pi$. The detailed solution to this equation system is documented in [28].

\section{B. Deriving the Energy Consumption Distribution}

Suppose at the beginning of a time unit $T^{u}$, the node is in state $i$ of $\left\{X_{n}\right\}$. Also suppose that during the time unit, the energy consumption by the node is $\varepsilon_{i}$, which is either measured, or is calculated according to the specifications of the hardware platform. An example will be given in Sec. V to show how to calculate $\varepsilon_{i}$. The $p d f^{1}$ of the energy consumption during the time unit is $g_{i}(e)=\delta\left(e-\varepsilon_{i}\right)$, where $\delta()$ is the delta function. Denote $h_{i j}^{(1)}(e)=g_{i}(e) q_{i j}$, where $q_{i j}$ is the $(i, j)$ th element of $Q_{X}$, the transition probability matrix for $\left\{X_{n}\right\}$ obtained in (7).

\footnotetext{
${ }^{1}$ It should be noted that, although a discrete time Markov process is used, the energy consumption is continuous in our analysis. Thus the $p d f$ is used to characterize the distribution.
} 
Then $h_{i j}^{(1)}(e)$ is the $p d f$ of energy consumption given that the Markov process transits from state $i$ to $j$.

For a given period $T$ during which the energy consumption distribution is to be obtained, without loss of generality, we assume that $T$ is measured in terms of the number of small time units of $T^{u}$. After $T$ time units $(T>1)$, the joint conditional $p d f$ becomes

$$
h_{i j}^{(T)}(e)=\sum_{k \in \mathcal{S}}\left(h_{i k}^{(1)} * h_{k j}^{(T-1)}\right)(e),
$$

where $\mathcal{S}$ is the set of all states in $\left\{X_{n}\right\}$. Therefore, if the matrix of $h_{i j}^{(T)}(e)$ is denoted as $\boldsymbol{H}^{(T)}(e)$, then $\boldsymbol{H}^{(T)}(e)$ is the $T$-fold convolution of $\boldsymbol{H}^{(1)}(e)$.

The energy consumption distribution during $T$ depends on the initial state of the system at the beginning of this period, which is usually randomly chosen. Thus the initial state probability vector is represented by the equilibrium state probability vector $\pi$. After $T$ time units, the $p d f$ and the $c d f$ of the energy consumption are

$$
f_{E(T)}(e)=\pi \boldsymbol{H}^{(T)}(e) \mathbf{1}, \quad F_{E(T)}(e)=\int_{0}^{e} f_{E(T)}(\epsilon) \mathrm{d} \epsilon,
$$

respectively, where $\mathbf{1}$ is the appropriately dimensioned column vector containing all 1 's.

\section{Asymptotic Energy Consumption Distribution}

If a QBD process is modeled by a DTMC, and each state in the DTMC is associated with a cost, then the sum of the total cost during a given period $T$ asymptotically approaches the Normal distribution [13]. Therefore, considering the energy consumption, $\varepsilon_{i}$, at each state $i$ as the cost, the total energy consumption during $T$, approaches the Normal distribution. The mean and the variance of the Normal distribution are given by

$$
\begin{aligned}
\mu(t) & =\boldsymbol{\pi} \varepsilon t=t \mu(1), \\
\sigma^{2}(t) & =\left(\sum_{i \in \mathcal{S}}\left(\varepsilon_{i}-\boldsymbol{\pi} \varepsilon\right)^{2} \pi_{i}+2 \boldsymbol{\beta} \varepsilon\right) t=t \sigma^{2}(1),
\end{aligned}
$$

respectively, where $\varepsilon$ is the vector of $\varepsilon_{i}, i \in \mathcal{S}$, and vector $\boldsymbol{\beta}$ is the solution of the following set of equations [13]:

$$
\boldsymbol{\beta}\left(\boldsymbol{Q}_{X}-\boldsymbol{I}\right)=-\gamma \boldsymbol{Q}, \quad \boldsymbol{\beta} \mathbf{1}=0,
$$

where $\gamma$ is a row vector whose $i$ th element is $\left(\varepsilon_{i}-\pi \varepsilon\right) \pi_{i}$.

\section{LifETIME DistRibution ANALYSis}

Using the $p d f$ of energy consumption $f_{E(T)}(e)$ in (9) for any given period $T$, the lifetime distribution of a node in a WSN can be found. The lifetime distribution for a given node, $L(C)$, is a function of its total battery capacity $C$. The probability that during $T$, the node consumes more energy than $C$ is

$$
\operatorname{Pr}(E(T)>C)=\int_{C}^{\infty} f_{E(T)}(e) \mathrm{d} e .
$$

This is exactly the probability that the lifetime of the node is shorter than $T$. Therefore the $c d f$ of the node lifetime is

$$
F_{L(C)}(t)=\operatorname{Pr}(L(C) \leq t)=\operatorname{Pr}(E(t)>C)=\int_{C}^{\infty} f_{E(t)}(e) \mathrm{d} e .
$$

As explained in Sec. III-C, when $t$ is large, $E(t) \sim$ $\mathcal{N}\left(\mu(t), \sigma^{2}(t)\right)$, where $\mu(t)$ and $\sigma^{2}(t)$ are given by (10) and (11), respectively. Thus, the $c d f$ of lifetime is approximated as

$$
F_{L(C)}(t) \approx Q\left(\frac{C-\mu(t)}{\sqrt{\sigma^{2}(t)}}\right) .
$$

The network lifetime, however, has different definitions according to applications and network topologies [5]. Whereas a complete investigation with various definitions is out of the scope of this paper, in this paper, we focus on the network lifetime defined as the duration before the battery depletion of the first node. Since every node needs to be alive during the network lifetime, the network lifetime $(N L)$ distribution is easily obtained for a WSN with $N$ nodes:

$$
F_{N L}(t)=\operatorname{Pr}(N L \leq t)=1-\prod_{i=1}^{N} \operatorname{Pr}\left(L_{i}\left(C_{i}\right) \geq t\right),
$$

where $L_{i}\left(C_{i}\right)$ is the lifetime for the $i$ th node with battery capacity $C_{i}$. Using the approximation given by (15) for the singlenode lifetime distribution, the network lifetime distribution is approximated by

$$
F_{N L}(t) \approx 1-\prod_{i=1}^{N}\left(1-Q\left(\frac{C_{i}-\mu_{i}(t)}{\sqrt{\sigma_{i}^{2}(t)}}\right)\right),
$$

where $\mu_{i}(t), \sigma_{i}^{2}(t)$ are given by (10) and (11) for the $i$ th node, respectively.

\section{CAse Study: Anycast PRotocol}

In this section, the techniques in Sec. III-IV to find the energy consumption and lifetime distributions are illustrated for a protocol with the anycast technique, which has been recently exploited in $[2,8,10,12,19]$. With the anycast technique, a node broadcasts beacon messages if it has packets to send. Then, one of responding neighbors is chosen as the next-hop node according to predefined rules (e.g., the first node that responds, or the closest node to the destination). Finally, the sender forwards the data packet to the chosen neighbor. While there is no single dominantly used anycast protocol in WSNs, in this paper, we model the following representative protocol.

A circular plane with a radius $R$ is considered, where nodes are located according to a Poisson distribution with density $\rho$. Sensor nodes report their readings to a sink, located at the center of the circular plane, through multi-hop routes in the network. The nodes (excluding the sink) turn off their radio transceivers periodically to save energy. When a node $A$ has a packet to send, it starts to repeatedly transmit RTS beacon packets in a CSMA/CA manner - the channel is sensed before the beacon transmission. If the channel is busy, a random backoff is performed and the channel is sensed again. When any other node $B$ in the transmission range is awake and hears the packet, it checks for the following criteria: 1$)$ node $B$ is closer to the sink than $A$, and 2) the signal-to-noise ratio (SNR) of the received RTS packet, $\psi$, is greater than some predefined threshold $\psi^{\text {th }}$. If both criteria are met, node $B$ sends a CTS packet. Node $A$ then chooses the first node that sent a CTS packet as the next-hop node and transmits the data packet to it. Successful data packet transmissions are acknowledged by the receiver, otherwise the sender retransmits the data packet until successful.

In order to reduce the waiting time for the packets spent in the queue, and balance the energy consumption in the network, we assume that each node responds to beacon packets only when it does not have packets to send. Considering the sink is awake all the time, if a node closer than a distance threshold $r^{t h}$ to the sink transmits beacons, it is assumed that no node except the sink will respond. Here $r^{t h}$ is chosen such that a high SNR is almost always guaranteed. Moreover, nodes are assumed to go to sleep when they finish transmitting all packets in the queue. As a result, compared to non-transmitting nodes, the active period is shorter. In cases where transmission energy consumption is significantly higher than listening, this helps balancing energy consumption among nodes. 


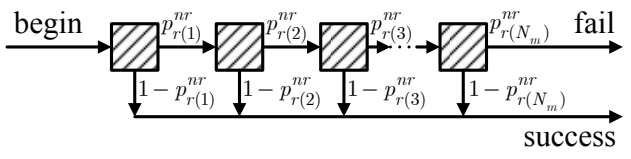

(a) $\left\{Z_{n}\right\}$

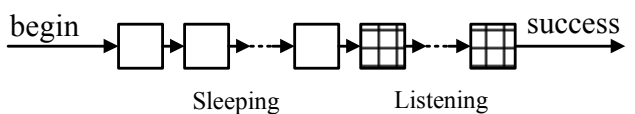

(b) $\left\{Y_{n}\right\}$

Fig. 2. The Markov chain structure of (a) the transmission process $\left\{Z_{n}\right\}$ and (b) the idle process $\left\{Y_{n}\right\}$ for the anycast protocol.

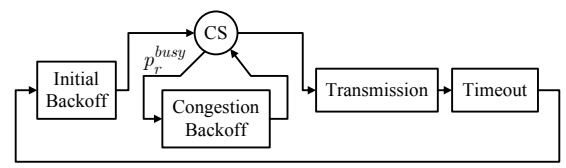

Fig. 3. The process of transmitting beacon packets.

The entire circular plane is discretized into concentric rings indexed by their distance to the sink, $r$. Each node senses the physical events, and generates packets with traffic rate $\lambda^{l}$. By symmetry, the relay traffic $\lambda_{r}^{f}$ is the same for all nodes in the same ring $r$. Each node has a queue length, $M$, a battery capacity, $C$, and operates in a duty cycle $\xi$.

In the following, we first show the DTMC $\left\{X_{n}\right\}$ for the protocol. Then, the protocol-specific parameters for the generic analysis in Sec. III, including the energy consumption at each state, and the transition probabilities for $\left\{X_{n}\right\}$ are derived. The energy consumption distribution for each node is obtained after these parameters are known. Finally, the lifetime distributions for each node and the network are found.

\section{A. Overview of the Markov Process}

The anycast protocol is modeled according to a DTMC $\left\{X_{n}\right\}$ discussed in Sec. III, which consists of multiple transmission blocks $\left\{Z_{n}\right\}$ and a single idle block $\left\{Y_{n}\right\}$. The structures of $\left\{Y_{n}\right\}$ and $\left\{Z_{n}\right\}$ for this protocol are shown in Fig. 2. $\left\{Y_{n}\right\}$ consists of two types of states chained together: sleeping states and listening states. When there is no packet arriving, the node enters these two parts of the chain alternately, mimicking the periodic sleeping and listening behavior. In the listening states, the node listens on the channel, thus both locally generated packets and relay packets can arrive. In the sleeping states, however, the node turns off its transceiver and only local packets can arrive. The number of states in $\left\{Y_{n}\right\}$ is $N^{f}=T^{s l} / T^{u}+T^{w} / T^{u}$, where $T^{s l}$ is the duration of the sleeping period, and $T^{w}$ is the duration of the listening period. A large $T^{u}$ can reduce the number of states in the DTMC, thus reducing computation cost for the model, but at the cost of reducing the granularity and accuracy of the result (Recall that we made the assumption that only one packet may arrive in a time unit. This is accurate only when $T^{u}$ is chosen small). When a packet arrives, the node terminates the idle process and begins the first layer of transmission process $\left\{Z_{n}\right\}$. In each $\left\{Z_{n}\right\}$ layer, the node keeps transmitting beacon packets. The number of states in $\left\{Z_{n}\right\}$ is $N^{m}=T^{m} / T^{u}$, where $T^{m}$ is the beacon time out.

At any time, a typical WSN node conducts one of the following communication tasks: transmission, listening, receiving, and sleeping. In this paper, we do not differentiate between listening and receiving, since most popular architectures, such

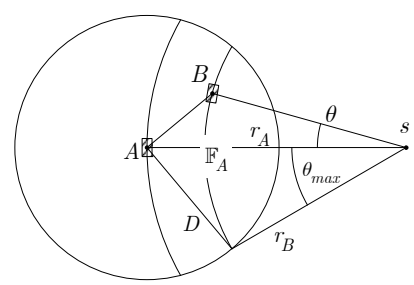

(a) Node $B$ is in the feasible region (b) Node $B$ is in the infeasible region of $A$.

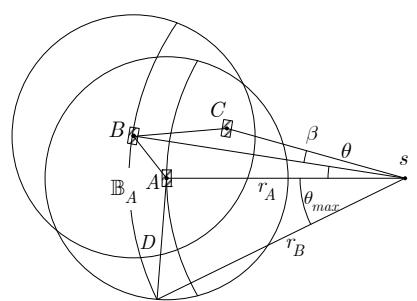
of $A$, and node $C$ is in the feasible region of $B$.
Fig. 4. Area around node $A$ is divided into small areas.

as Mica2 [23] and TelosB [25], consume similar power when listening or receiving. We also ignore the energy consumed by the data packet transmission. This is a valid simplification, because usually the majority of energy is consumed by idle listening and beacon transmissions. Therefore, there are 3 types of states in $\left\{X_{n}\right\}$ : Beacon transmission, Sleeping, and Listening states. Nodes consume a specific amount of energy $\varepsilon$ in states of each type, as will be discussed in the following.

An additional assumption made in this case study is to ignore the hidden terminals. For high density networks, hidden terminal effects can be easily captured by the model as we showed in [28]. Testbed and simulation results in Sec. VI suggest that, with these assumptions, the derived energy consumption distribution still agrees well with empirical results.

\section{B. Energy Consumption in Each State}

In practice, since battery voltage drops over time, battery capacity is often measured with normalized voltage. Therefore, in this paper, energy is represented in the units of A.sec. In sleeping and listening states, the energy consumed during a time unit, $T^{u}$, are $\varepsilon^{\text {sleep }}=I^{\text {sleep }} T^{u}$, and $\varepsilon^{\text {listen }}=I^{\text {listen }} T^{u}$, respectively, where $I^{\text {sleep }}$ and $I^{\text {listen }}$ are the measured current drawn from the battery in the sleep and listening modes, respectively.

The power consumption when the node is transmitting beacon packets, $\varepsilon^{\text {beacon }}$, however, depends on the beacon transmission process shown in Fig. 3. For every beacon packet, the node waits for a uniformly distributed random initial backoff with a maximum duration $T_{\text {max }}^{i b o}$, and whenever the channel is sensed busy before transmission, a congestion backoff is performed. The congestion backoff is also uniformly distributed with a maximum duration $T_{m a x}^{c b o}$. These backoffs are used to avoid constant beacon packets collision among nodes. Then, the transmission takes a duration of $T^{t x}$, which is determined by the packet size and the data rate. Finally, after the transmission, a timeout period of $T^{t o}$ is spent to wait for any possible CTS response. Therefore, the node transmits beacons only in a portion of time, and the portion, $\omega^{\text {beacon }}$, should be obtained first to determine $\varepsilon^{\text {beacon }}$. For a node $A$ within $\operatorname{ring} r_{A}, \omega^{\text {beacon }}$ is expressed as

$\omega_{r_{A}}^{\text {beacon }}=T^{t x} /\left(T_{\max }^{i b o} / 2+T_{\max }^{c b o} / 2\left(1-p_{r_{A}}^{b u s y}\right)+T^{t x}+T^{t o}\right)$

where $p_{r_{A}}^{\text {busy }}$ is the probability of sensing the channel busy, and is derived as follows.

First, as shown in Fig. 4(a), the region within the transmission range of $A, \mathbb{N}_{A}$, is divided into small areas according to the polar coordinates centered at the sink. Each small area has a size approximated by $\Delta r \Delta \theta r$. Then, the probability of sensing the channel busy, $p_{r_{A}}^{\text {busy }}$, is the probability that there is at least one node transmitting a packet in these areas. Considering that in WSN applications, sleeping cycles are usually long and duty cycles are usually very small, a sender node often has to 
wait for a relatively long period transmitting beacon packets before receiving a CTS response. Therefore, beacon packets are considered the dominant packets in the channel, and the major reason of a busy channel [8]. Thus, in the small area $(r: r+\Delta r, \theta: \theta+\Delta \theta)$, denote $p_{r}^{e}$ as the probability that there exists a node in this area, and $\phi_{r}^{\text {Beacon }}$ as the probability that at any time a node in this area, if it exists, is transmitting a beacon packet. Then $p_{r_{A}}^{\text {busy }}$ is given $\mathrm{by}^{2}$

$$
p_{r_{A}}^{\text {busy }}=1-\prod_{r=r_{A}-D}^{r_{A}+D} \prod_{\theta=-\theta_{\max }}^{\theta_{\max }}\left(1-p_{r}^{e} \phi_{r}^{\text {Beacon }}\right),
$$

where $D$ is the radius of transmission range of each node, $\theta_{\max }$ is given by $\theta_{\max }=\cos ^{-1}\left(\left(r_{A}^{2}+r^{2}-D^{2}\right) / 2 r_{A} r\right)$. By symmetry, $p_{r}^{e}$ is the same for all areas in ring $r$ regardless of $\theta$. In this small area, $p_{r}^{e}$ is approximated by

$$
p_{r}^{e}=e^{-\rho \Delta r \Delta \theta r} \rho \Delta r \Delta \theta r \approx \rho \Delta r \Delta \theta r,
$$

where $\rho$ is the density parameter of the Poisson node distribution. The probability that a node in this area is transmitting a beacon packet, $\phi_{r}^{\text {Beacon }}$, is given by $\phi_{r}^{\text {beacon }}=\pi_{r}^{\text {beacon }} \omega_{r}^{\text {beacon }}$, where $\pi_{r}^{\text {beacon }}$ is the total probability that the node is in one of the beacon transmission states in the DTMC $\left\{X_{n}\right\}$, and is given by adding the probabilities in the equilibrium state probability vector, $\boldsymbol{\pi}_{r}$, (which will be discussed in Sec. V-C) corresponding to the beacon transmission states. Therefore, $\omega_{r_{A}}^{\text {beacon }}$ for node $A$ depends on its values for other nodes in $A$ 's neighborhood, $\mathbb{N}_{A}$. An iterative procedure is used to calculate $\omega_{r}^{\text {beacon }}$ for all $r$ 's at the end of Sec. V. Then, $\varepsilon^{\text {beacon }}$ is obtained by

$$
\varepsilon^{\text {beacon }}=\left(I^{\text {listen }}\left(1-\omega_{r}^{\text {beacon }}\right)+I^{t x} \omega_{r}^{\text {beacon }}\right) T^{u},
$$

where $I^{t x}$ is the measured transmission current.

\section{Constructing the DTMC $\left\{X_{n}\right\}$}

The other parameters in $\left\{X_{n}\right\}$, i.e., transition probability matrices in $\left\{Y_{n}\right\}$ and $\left\{Z_{n}\right\}$, are obtained according to the Markov structure in Fig. 2. In either $\left\{Y_{n}\right\}$ or $\left\{Z_{n}\right\}$, there is only one initial state (denoted by "begin"), with the initial probability of 1. From each state, if there is an outgoing transition denoted as "success" or "fail", it has a probability to complete the current transmission or idle process in a success or failure, respectively. The transition probabilities among states are shown in Fig. 2. Note that transitions with a probability of 1 are not labeled. The transition probabilities $p_{r_{A}(i)}^{n r}$, and the traffic rate $\lambda_{Y}, \lambda_{Z}$ are explained in the following.

In the $i$ th time unit in $\left\{Z_{n}\right\}$, a node $A$ in ring $r_{A}$ has a probability of $p_{r_{A}(i)}^{n r}$ of not receiving any CTS response, and enters the next state. If in all $N_{m}$ states, node $A$ receives no CTS response, the transmission fails and the packet is dropped. On the other hand, if in any of the states, a CTS response is received, the node transmits the packet and the transmission succeeds. The probability $p_{r_{A}(i)}^{n r}$ is the conditional probability that given the transmissions in the previous $i-1$ states failed, the transmissions in the $i$ th state still fails. Therefore,

$$
\begin{aligned}
& p_{r_{A}(1)}^{n r}=p_{r_{A}(1 \sim 1)}^{n r} \\
& p_{r_{A}(i)}^{n r}=p_{r_{A}(1 \sim i)}^{n r} / p_{r_{A}(1 \sim i-1)}^{n r}, 2 \leq i \leq N^{m}
\end{aligned}
$$
$\prod_{r=r_{A}-D}^{2}$ With a slight abuse of notations, for the sake of space and clarity, we use
also applies to other products and sums of lengths and angles for small area analyses in the remaining part of the paper. where $p_{r_{A}(1 \sim i)}^{n r}$ is the probability that during all first $i$ states in $\left\{Z_{n}\right\}$, beacon transmission fails, since no CTS packet is received in these states. Therefore,

$$
p_{r_{A}(1 \sim i)}^{n r}=\prod_{r_{B}=r_{A}-D}^{r_{A}-1} \prod_{\theta=-\theta_{\max }}^{\theta_{\max }}\left(1-p_{r_{B}}^{e} p_{r_{B}(i)}^{o l} p_{A, B, \theta}^{S N R}\right),
$$

where each of the small areas, $\left(r_{A}: r_{A}+\Delta r, \theta: \theta+\Delta \theta\right)$, is located within the transmission range of $A, \mathbb{N}_{A}$, and is closer to the sink than $A$ (this range is called the feasible region of $A, \mathbb{F}_{A}$, as shown in Fig. 4(a)); $r_{B}$ is the distance from the small area to the sink. $\theta_{\max }$ is given by $\theta_{\max }=$ $\cos ^{-1}\left(\left(r_{A}^{2}+r_{B}^{2}-D^{2}\right) / 2 r_{A} r_{B}\right) ; p_{r_{B}}^{e}$ is the probability that there exists a node in each area, and is given by $(20) ; p_{r_{B}(i)}^{o l}$ is the probability that the waking period of a node $B$, which is located $r_{B}$ away from the sink, overlaps with the first $i$ beacon transmission time units of $A$; and $p_{A, B}^{S N R}$ is the probability that a packet, transmitted from node $A$ to node $B$ with polar coordinates $\left(r_{B}, \theta\right)$ to the sink, has an SNR higher than some predefined threshold $\psi^{t h}$. It is obtained by Eq. (10) in [30].

The probability that the waking period of $B$ overlaps with the first $i$ beacon transmission time units of $A, p_{r_{B}(i)}^{o l}$, is derived as follows. If node $A$ receives no response in each of the small areas, at least one of the following statements is true: 1) a node does not exist in the area, 2) at least one node exists but they are sleeping during any of the first $i$ slots, and 3) at least one node exists and is awake, but the SNR of the beacon packet they receive is lower than the predefined threshold $\psi^{t h}$. Node $B$ is awake during any of the first $i$ slots means that the first beacon transmission time unit of $A$ either coincides with any of the awake time units of $B$ or coincides with the last $i-1$ sleeping units of $B$. Thus, $p_{r_{B}(i)}^{o l}$ is given by

$$
p_{r_{B}(i)}^{o l}=\sum_{j=1}^{N^{w}} \boldsymbol{\pi}_{r_{B}}^{W_{j}}+ \begin{cases}\sum_{j=N^{s}-i+1}^{N^{s}} \boldsymbol{\pi}_{r_{B}}^{S_{j}}, & 1 \leq i<N^{s} \\ \sum_{j=1}^{N^{s}} \pi_{r_{B}}^{S_{j}}, & i \geq N^{s}\end{cases}
$$

where $N^{s}=N^{f}-N^{w}$ is the number of sleeping time units in $\left\{Y_{n}\right\}, \boldsymbol{\pi}_{r_{B}}^{W_{j}}$ and $\boldsymbol{\pi}_{r_{B}}^{S_{j}}$ are the equilibrium probability that $B$ is in the $j$ th awake state or sleeping state in $\left\{X_{n}\right\}$, respectively. $N^{f}$ and $N^{w}$ are the number of total states and awake states in $\left\{Y_{n}\right\}$, respectively.

Therefore, $p_{r_{A}(1 \sim i)}^{n r}$ in (23) is determined using (20) -(24), and $p_{r_{A}(i)}^{n r}$ in (22) is obtained using (23).

Next, the traffic rate at each state, $\boldsymbol{\lambda}_{I}$ and $\boldsymbol{\lambda}_{L}$, are discussed. The arriving traffic at $A$ contains locally generated and relay traffic. In sleeping states, the traffic arrival rate is $\lambda^{l}$. In listening states, the traffic rate is $\lambda_{r_{A}}^{f}+\lambda_{r_{A}}^{l}$. Finally, in the beacon transmission states, since nodes are assumed not to respond to any relay packets, the traffic rate is $\lambda^{l}$.

Consider the small area $\left(r_{A}: r_{A}+\Delta r, \theta: \theta+\Delta \theta\right)$, where the forwarded traffic arrives from any node $B$ in the infeasible region $\mathbb{B}_{A}=\mathbb{N}_{A} \backslash \mathbb{F}_{A}$, as shown in Fig. 4(b). Therefore $\lambda_{r_{A}}^{f}$ is given by

$$
\lambda_{r_{A}}^{f}=\frac{\sum_{r_{B}=\max \left(D+1, r_{A}+1\right)}^{r_{A}+D} \sum_{\theta=-\theta_{\max }}^{\theta_{\max }} p_{r_{B}}^{e} \lambda_{r_{B}}^{o} p_{B, A}^{f w}}{p_{r_{A}}^{e} \pi_{r_{A}}^{\text {listen }}},
$$

where $\lambda_{r_{B}}^{o}$ is the output traffic transmitted from $B . \pi_{r_{A}}^{\text {listen }}$ is the probability that $A$ is in any listening state, and is the sum of the probabilities corresponding to all listening states in $\boldsymbol{\pi}_{r_{A}}$. Moreover, $\lambda_{r_{B}}^{o}$ is calculated by

$$
\lambda_{r_{B}}^{o}=\boldsymbol{\lambda}_{r_{B}}\left(\boldsymbol{\pi}_{r_{B}}\right)^{\mathrm{T}}\left(1-p_{r_{B}}^{q \text { full }}-p_{r_{B}}^{\text {drop }}\right),
$$


where $p_{B, A}^{f w}$ is the probability that a node $B$ forwards a packet to node $A$, among all possible forward targets, and $\boldsymbol{\lambda}_{r_{B}}$ is the traffic rate vector for all states in $\left\{X_{n}\right\}$ for $B$. The probability that the packet is dropped due to beacon transmission timeout, $p_{r_{B}}^{d r o p}$, is easily obtained as $p_{r_{B}}^{d r o p}=p_{r_{B}\left(1 \sim N_{r_{B}}^{m}\right)}^{n r}$ (see (23)). The probability that the queue is full when the packet arrives, $p_{r_{B}}^{q f u l l}$, is obtained by $p_{r_{B}}^{q f u l l}=\boldsymbol{\pi}_{r_{B}}^{M} \boldsymbol{A}_{u} \mathbf{1}$, where $\boldsymbol{\pi}_{r_{B}}^{M}$ is the probability vector corresponding to the layer $M$ in $\boldsymbol{\pi}_{r_{B}}$, and $\boldsymbol{A}_{u}$ is given by (1) for node $B$. In (25), $p_{B, A}^{f w}$ is proportional to the probability that $A$ is available when $B$ transmits a beacon, and is normalized on the total probability of available for all possible nodes. The probability of availability is given by

$$
p_{B, A}^{\text {avail }}=p_{r_{A}}^{e} p_{r_{A}}^{\text {wake }} p_{B, A}^{S N R}
$$

where $p_{r_{A}}^{\text {wake }}=\sum_{j=1}^{N^{w}} \boldsymbol{\pi}_{r_{A}}^{W_{j}}$ is the probability that node $A$ is awake, and $\pi_{r_{A}}^{W_{j}}$ is the equilibrium probability that $A$ is in the $j$ th waking state in $\left\{X_{n}\right\}$. Then, $p_{B, A}^{f w}$ in (25) is calculated as

$$
p_{B, A}^{f w}=\frac{p_{B, A}^{\text {avail }}}{\sum_{r_{C}=r_{B}-D}^{r_{B}-1} \sum_{\beta=\theta-\beta_{\max }}^{\theta+\beta_{\max }} p_{B, C}^{\text {avail }}},
$$

where node $C$, with the polar coordinates $\left(r_{C}, \beta\right)$, can be in any small area in $\mathbb{F}_{B}$.

Thus, according to (25), the traffic rate of node $A$ at each state is determined. Then, the equilibrium state probability for the DTMC $\left\{X_{n}\right\}, \pi_{r_{A}}$ is obtained. Note that thus far, while we solve $\omega_{r_{A}}^{\text {beacon }}$, it is assumed that $\omega_{r}^{\text {beacon }}$ for all nodes in range are known. This circular dependency is solved using an iterative manner: first, arbitrary initial guesses of $\omega_{r}^{\text {beacon }}$ for all rings are used, then updated values of $\omega_{r}^{\text {beacon }}$ are calculated. The iteration terminates when the difference of these variables between two consecutive iterations are negligible for each ring. Then, $\varepsilon_{r}^{\text {beacon }}$, the energy consumption during a beacon time unit is obtained by (21). Accordingly, the energy consumption distribution for any single node is calculated by (9).

With the energy consumption distribution for nodes in each ring known, the lifetime distribution for nodes in each ring, $L_{r}(C)$, is directly obtained by (15). Then, the distribution of the network lifetime, and its Normal distribution approximation are

$$
\begin{aligned}
\operatorname{Pr}(N L \leq t) & =1-\prod_{r=0}^{R} \prod_{\theta=-\pi}^{\pi}\left(1-p_{r}^{e} \operatorname{Pr}\left(L_{r}(C) \leq t\right)\right) \\
& \approx 1-\prod_{r=0}^{R} \prod_{\theta=-\pi}^{\pi}\left(1-p_{r}^{e} Q\left(\frac{C-t \mu_{r}(t)}{\sqrt{\sigma_{r}^{2}(t)}}\right)\right)
\end{aligned}
$$

where $\mu_{r}(t)$ and $\sigma_{r}^{2}(t)$ are given by (10) and (11) for nodes in ring $r$, respectively. Note that since we adopted a probabilistic topology, these equations are slightly varied from (16) and (17).

\section{Extension to Other Protocols}

The techniques in this section for the anycast protocol can be used to obtain the energy consumption and the lifetime distribution for other protocols, for example, TDMA protocols and XMAC[3]. First, the Markov chains $\left\{X_{n}\right\}$ is constructed according to the specific protocol behavior. Then, the single-node energy consumption distribution is obtained by (9). Finally, the single-node and network lifetime distributions are found using
(15) and (16), (17), respectively. The detailed solutions for other protocols are left in our future work for space considerations.

\section{ANALYTICAL RESUltS AND EMPIRICAL VALIDATIONS}

The energy consumption distribution model has been evaluated using MATLAB to determine the distribution for singlenode energy consumption, single-node lifetime, and the network lifetime for the anycast MAC protocol. The computing environment is a computer with a Xeon $5150 \mathrm{CPU}$ working at $2.66 \mathrm{GHz}$ and $2 \mathrm{G}$ RAM. For any network setup in the experiments below, the calculation for the asymptotic Normal distribution of the energy consumption during any given duration takes less than 1 minute. In contrast, the TOSSIM-based simulations [11] determine the energy consumption distribution in the same order of simulated time, and is significantly slower. For example, for a simulated duration of 2 hours, the simulation takes roughly 30 mins. In this section, we show that our model also provides a high accuracy against both empirical experiments and simulations.

\section{A. Validation of the Single-node Energy Analysis}

We first show that the analytical prediction of energy consumption distribution in (9) matches with the empirical experiments. Our testbed consists of 28 Crossbow TelosB motes. The nodes are randomly placed in a circular area of radius $R=4 \mathrm{~m}$. The data packet size is $l_{p}=40$ bytes, whereas the beacon message and the CTS response message have the same size of $l_{m}=22$ bytes. Each node generates the same amount of local traffic, and send packets towards the sink according to a Bernoulli process with average rate $\lambda^{l}=0.0042$ in each time unit $T^{u}=0.25 \mathrm{sec}$, which equals to 1 packet per minute. The sleeping cycle of each node is $T^{f}=10 \mathrm{sec}$, during which the wake period is $T^{w}=5 \mathrm{sec}$, thus the duty cycle is $\xi=0.5$. Moreover, the beacon transmission timeout is $T^{m}=10 \mathrm{sec}$. The maximum duration of initial backoff and congestion backoff are $T_{\max }^{i b o}=10 \mathrm{msec}$ and $T_{\max }^{c b o}=2.5 \mathrm{msec}$, respectively. The beacon transmissions duration is $T^{t x}=1.6 \mathrm{msec}$ and the interbeacon timeout is $T^{t o}=12 \mathrm{msec}$. The transmission power is set to $-15 \mathrm{dBm}$ for all nodes. Other parameters for the channel are: $P_{n}=-105 \mathrm{dBm}, P_{L}\left(D_{0}\right)=52.1 \mathrm{~dB}, D_{0}=1 \mathrm{~m}, \eta=3.3$, $\sigma^{s}=5.5$. The threshold radius $r^{t h}$ is set to $2.5 \mathrm{~m}$, within which all nodes only transmit packets to the sink. The SNR threshold is set to $\psi^{t h}=10 \mathrm{~dB}$. The experiment is conducted for 24 hours, and the current drawn from the battery is continuously measured and logged using NI-USB 6210 DAQ modules [24]. The energy consumption distributions during $T=60$ s for two nodes, which have a distance of $2.6 \mathrm{~m}$ and $3.5 \mathrm{~m}$ to the sink, respectively, are measured. The $c d f$ s of the measured energy consumption are shown in Fig. 5, along with the prediction by our analytical model (see (9)). It can be observed that the predictions matches well with the empirical measurements for each node.

The same network topology is also simulated using TOSSIM. The results of the energy consumption distribution for each of the two nodes are also shown in Fig. 5. The results suggest that both simulation and our analytical model produce accurate prediction of the empirical distribution. Therefore, in further experiments, we use simulation results to validate our model for larger scale networks, and for a longer duration.

For longer durations, i.e., $T=10$ and $T=20$ minutes, the $c d f$ of energy consumption for node at $r=3.5 \mathrm{~m}$ are given in Fig. 6. The $c d f$ of the asymptotic Normal distributions predicted by our model (see (10) and (11))are also shown. It can be observed that as the duration increases, the energy consumption 
distribution converges to the asymptotic Normal distribution. We further validate the accuracy of the asymptotic approximation using Kurtosis [7, 26] as follows.

\section{B. Validation of the Normal Distribution Approximation}

To reveal the similarity between the energy consumption distribution and the asymptotic Normal distribution, Kurtosis is often used. For a random variable $x$, Kurtosis is defined as $\kappa(x)=\mu_{4}(x) / \sigma^{4}(x)$, where $\mu_{4}(x)$ is the fourth moment of $x$, and $\sigma(x)$ is the standard deviation. The closer $\kappa(x)$ is to 3 , the closer $x$ is to a Normal distributed variable. In the following simulations, a network in a circular area is assumed, and the topology is randomly generated according to a Poisson distribution with density $\rho=0.05$. Each topology is simulated for 48 hours, and 100 different topologies are generated. The energy consumption during $1,2,4, \ldots, 128$ minutes for a node at $r=16 \mathrm{~m}$ are recorded to evaluate the Kurtosis. The parameters used are as follows: network radius $R=20 \mathrm{~m}$, traffic rate is $0.1 \mathrm{pkt} / \mathrm{min}, r^{t h}=10 \mathrm{~m}$, duty cycle is 0.2 , and all other parameters remain the same as in the previous experiments. The result shown in Fig. 7(a) reveals that when the duration $T$ increases to above $16 \mathrm{mins}$, the Kurtosis of the energy consumption converges to 3 , which suggests that its distribution converges to the Normal distribution. The predicted mean and variance of energy consumption are shown in Fig. 7(b) and are compared to the simulation. The results show that the mean and the variance increase linearly with the duration, and is almost perfectly predicted by our model.

\section{Relationships between Lifetime and Network Parameters}

Next, using the lifetime distribution predicted by (15), we investigate the relationship between the probability of achieving a given node or the network lifetime, and various network parameters. In each of the following tests, the network density $\rho$, the duty cycle $\xi$ for all nodes, and the traffic rate $\lambda^{l}$ for all nodes are varied, respectively. The default values for these parameters are $0.052,0.2$, and $0.1 \mathrm{pkt} / \mathrm{min}$, respectively. Other parameters are kept unchanged from the previous experiment. The battery capacities for all nodes are $C_{r}=2000 \mathrm{~mA} \cdot \mathrm{H}$. The probability that the lifetime of a node at distance $r=12 \mathrm{~m}$ is longer than 500 hours is shown in Fig. 8. The results reveal that for the maximum probability of achieving this lifetime, the density should be no less than 0.053 . This is because if the density is low, when transmitting beacon packets, each node needs to wait for a longer time before other nodes in the feasible region wake up, thus consuming more energy. Moreover, reducing duty cycle directly reduces the energy consumption, as observed in Fig. 8. Finally, either increasing or reducing the traffic rate from $0.05 \mathrm{pkt} / \mathrm{min}$ results in a decrease of the probability of achieving this lifetime. This is because in the protocol, when each node is transmitting beacon packets, it does not respond to other beacon packets. Therefore, with a higher traffic rate, more nodes are transmitting, and fewer are available to send CTS responses. Thus transmitting nodes need to wait for a longer time, and the energy spent on transmitting is higher. With a moderate traffic rate, available relay nodes are enough, transmitting nodes can finish their transmissions and go to sleep early, effectively saving energy. With a lower traffic rate, however, the chance that nodes relay a packet and go to sleep early is reduced, thus consuming more energy than with a moderate traffic rate.

In the same network settings, the network lifetime distribution is also examined. The probability of achieving a 500 hour network lifetime (see (15)) is shown in Fig. 8(d) for various

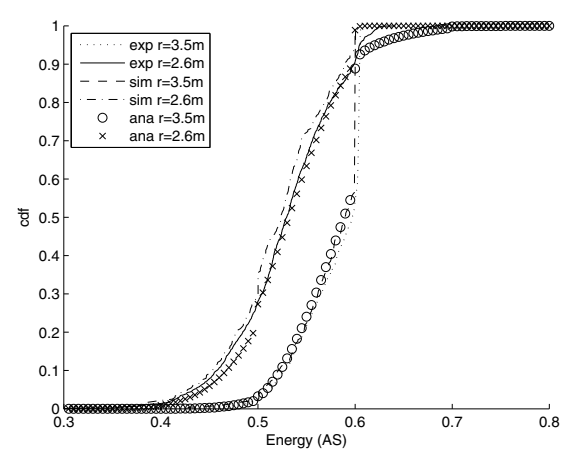

Fig. 5. $c d f$ of the energy consumption during $1 \mathrm{~min}$. Empirical experiments, simulation results, and analytical prediction are shown.

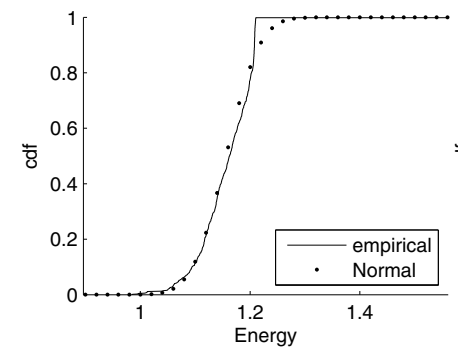

(a) $T=2 \mathrm{~s}$

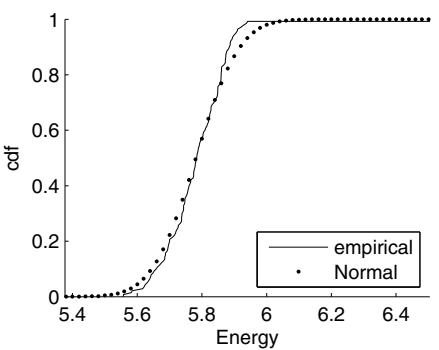

(b) $T=10 \mathrm{~s}$
Fig. 6. $c d f$ of the energy consumption during longer periods. As the duration increases, the energy consumption approaches the asymptotic Normal distribution.

network densities. To achieve this lifetime, the optimal density is greater than 0.097 , which is significantly higher than the value for a single node lifetime guarantee in Fig. 8(a). This is because the network is only alive when all nodes are alive, which is a much stronger requirement than for a single alive node.

\section{CONCLUSION AND FUtURE WORK}

In this paper, a probabilistic analysis of the energy consumption in a random multi-hop network is provided. A discrete time Markov chain is used to model the transmission process, and predict the energy consumption distribution for each node. It is shown that, when the time duration is long, the energy consumption converges to a Normal distribution, and the mean and variance of such distribution are also provided. With the help of energy consumption distribution, the lifetime distributions for each node and the entire network are derived. The developed model is validated by both testbed experiments and TOSSIM simulations. The results show that the developed framework accurately models the distribution of the energy consumption and captures the randomness of multi-hop WSNs.

As future work, we plan to analyze the energy consumption for more MAC protocols, such as BMAC [21], XMAC [3], using our model. We also plan to extend the model to capture more generic network topologies, and traffic types, such as periodic and bursty traffics. Moreover, other network lifetime definitions will be investigated.

\section{REFERENCES}

[1] K. Akkaya and M. Younis, "A survey on routing protocols for wireless sensor networks," Ad Hoc Networks, vol. 3, pp. 325-349, 2005.

[2] I. Akyildiz, M. Vuran, and O. Akan, "A cross-layer protocol for wireless sensor networks," in Information Sciences and Systems, 2006 40th Annual Conference on, March 2006, pp. 1102-1107. 


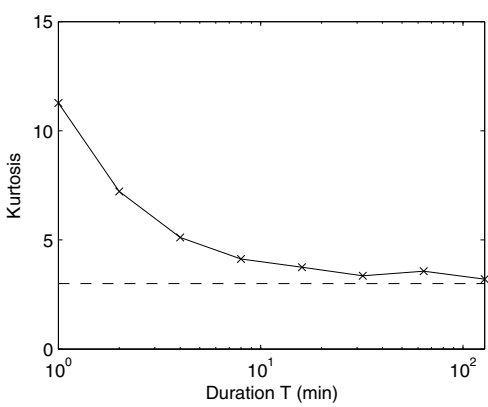

(a) Kurtosis

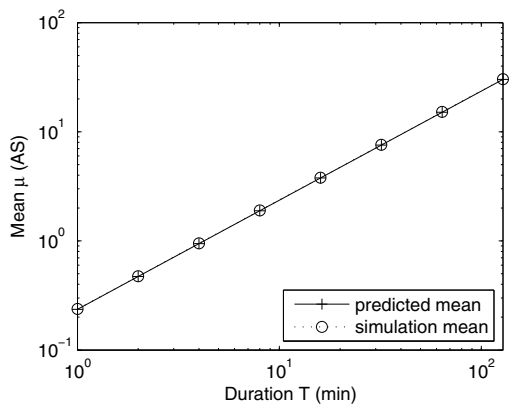

(b) Mean

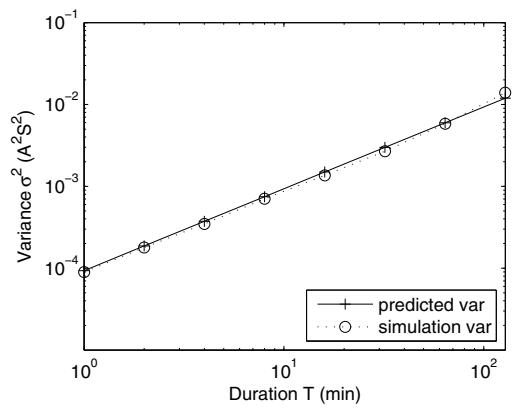

(c) Variance

Fig. 7. Energy consumption during a long period converges to the Normal distribution.

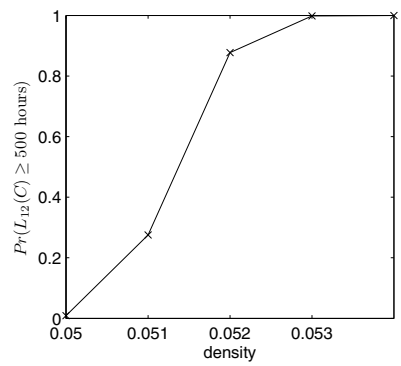

(a) Density varies

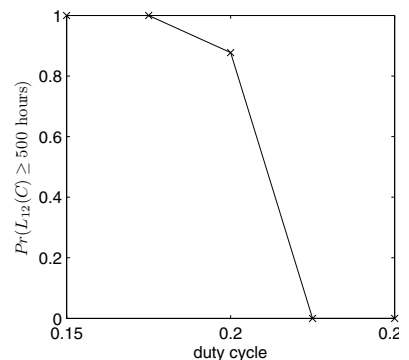

(b) Duty cycle varies

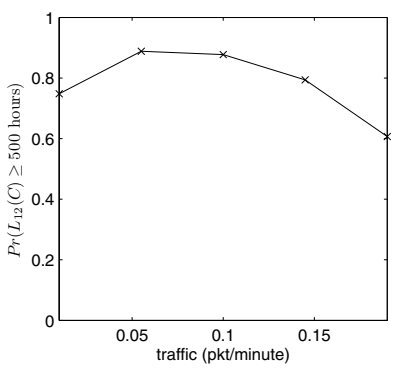

(c) Traffic rate varies

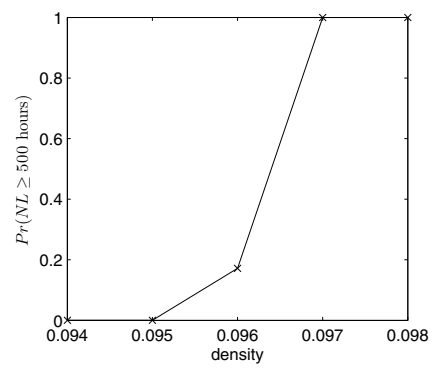

(d) Density varies

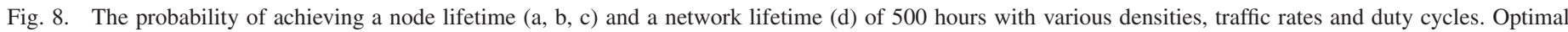
ranges of parameters are suggested for maximum probability of achieving this lifetime.

[3] M. Buettner, G. V. Yee, E. Anderson, and R. Han, "X-MAC: a short preamble MAC protocol for duty-cycled wireless sensor networks," in SenSys '06: Proceedings of the 4th international conference on Embedded networked sensor systems. New York, NY, USA: ACM, 2006, pp. 307320.

[4] I. Demirkol, C. Ersoy, and F. Alagoz, "MAC protocols for wireless sensor networks: a survey," Communications Magazine, IEEE, vol. 44, no. 4, pp. 115-121, 2006.

[5] I. Dietrich and F. Dressler, "On the lifetime of wireless sensor networks," ACM Trans. Sen. Netw., vol. 5, no. 1, pp. 1-39, 2009.

[6] J. Haapola, Z. Shelby, C. Pomalaza-Raez, and P. Mahonen, "Cross-layer energy analysis of multihop wireless sensor networks," in Wireless Sensor Networks, 2005. Proceeedings of the Second European Workshop on, Jan.2 Feb. 2005, pp. 33-44

[7] A. Hyvarinen, J. Karhunen, and E. Oja, Independent Component Analysis. Wiley-Interscience, May 2001

[8] X. L. Joohwan Kim and N. Shroff, "Optimal anycast technique for delaysensitive energy-constrained asynchronous wireless sensor networks," INFOCOM 2009. The 28th Conference on Computer Communications. IEEE, pp. 412-421, Apr. 2009

[9] D. Jung, T. Teixeira, and A. Savvides, "Sensor node lifetime analysis: Models and tools," ACM Trans. Sen. Netw., vol. 5, no. 1, pp. 1-33, 2009

[10] S. Kulkarni, A. Iyer, and C. Rosenberg, "An address-light, integrated mac and routing protocol for wireless sensor networks," Networking, IEEE/ACM Transactions on, vol. 14, no. 4, pp. 793-806, Aug. 2006.

[11] P. Levis, N. Lee, M. Welsh, and D. Culler, "TOSSIM: accurate and scalable simulation of entire tinyos applications," in SenSys '03: Proceedings of the 1st international conference on Embedded networked sensor systems. New York, NY, USA: ACM Press, 2003, pp. 126-137.

[12] S. Liu, K.-W. Fan, and P. Sinha, "CMAC: An energy efficient mac layer protocol using convergent packet forwarding for wireless sensor networks," in Sensor, Mesh and Ad Hoc Communications and Networks, 2007. SECON '07. 4th Annual IEEE Communications Society Conference on, June 2007, pp. 11-20.

[13] Y. Nazarathy and G. Weiss, "The asymptotic variance rate of the output process of finite capacity birth-death queues," Queueing Systems, vol. 59, no. 2, pp. 135-156, June 2008.

[14] R. Nelson, Probability, stochastic processes, and queueing theory: the mathematics of computer performance modeling. New York, NY, USA: Springer-Verlag New York, Inc., 1995.

[15] M. F. Neuts, Matrix-Geometric Solutions in Stochastic Models: an Algorthmic Approach. Dover Publications Inc., 1981.

[16] - Structured Stochastic Matrices of M/G/1 Type and Their Applica- tions. Marcel Dekker, 1989

[17] M. Noori and M. Ardakani, "A probabilistic lifetime analysis for clustered wireless sensor networks," in Wireless Communications and Networking Conference, 2008. WCNC 2008. IEEE, 31 2008-April 3 2008, pp. 23732378.

[18] _ _ "A probability model for lifetime of event-driven wireless sensor networks," in Sensor, Mesh and Ad Hoc Communications and Networks, 2008. SECON '08. 5th Annual IEEE Communications Society Conference on, June 2008, pp. 269-277.

[19] W. Pak, J.-G. Choi, and S. Bahk, "Tier based anycast to achieve maximum lifetime by duty cycle control in wireless sensor networks," in Wireless Communications and Mobile Computing Conference, 2008. IWCMC '08. International, Aug. 2008, pp. 123-128.

[20] P. Park, P. Di Marco, P. Soldati, C. Fischione, and K. Johansson, "A generalized markov chain model for effective analysis of slotted IEEE 802.15.4,' in Mobile Adhoc and Sensor Systems, 2009. MASS '09. IEEE 6th International Conference on, Oct. 2009, pp. $130-139$.

[21] J. Polastre, J. Hill, and D. Culler, "Versatile low power media access for wireless sensor networks," in SenSys '04: Proceedings of the 2nd international conference on Embedded networked sensor systems. New York, NY, USA: ACM, Nov 2004, pp. 95-107.

[22] R. Shah and J. Rabaey, "Energy aware routing for low energy ad hoc sensor networks," in Wireless Communications and Networking Conference, 2002. WCNC2002. 2002 IEEE, vol. 1, Mar 2002, pp. 350-355 vol.1.

[23] "MICA2 sensor node," web, http://www.xbow.com.

[24] “NI USB-6210 multifunction DAQ," web, http://www.ni.com.

[25] “TelosB sensor node," web, http://www.xbow.com.

[26] S. Vural and E. Ekici, "Probability distribution of multi-hop-distance in one-dimensional sensor networks," Comput. Netw., vol. 51, no. 13, pp. 3727-3749, 2007.

[27] Q. Wang, M. Hempstead, and W. Yang, "A realistic power consumption model for wireless sensor network devices," in Sensor and Ad Hoc Communications and Networks, 2006. SECON'06. 2006 3rd Annual IEEE Communications Society on, vol. 1, Sept. 2006, pp. 286-295.

[28] Y. Wang, M. C. Vuran, and S. Goddard, "Cross-layer analysis of the endto-end delay distribution in wireless sensor networks," in Proc. IEEE RTSS '09, Washington, DC, Dec 2009.

[29] W. Ye, J. Heidemann, and D. Estrin, "Medium access control with coordinated adaptive sleeping for wireless sensor networks," IEEE/ACM Trans. Netw., vol. 12, no. 3, pp. 493-506, 2004

[30] M. Zúniga and B. Krishnamachari, "An analysis of unreliability and asymmetry in low-power wireless links," ACM Trans. Sen. Netw., vol. 3, no. 2, June 2007. 